\title{
Detection of Breast Pathology using Thermography as a Screening Tool
}

by Aayesha Hakim*, R.N. Awale**

\author{
*Veermata Jijabai Technological Institute, Mumbai, India, aayesha.hakim@gmail.com \\ **Veermata Jijabai Technological Institute, Mumbai, India, rnawale@el.vjitiac.in
}

\begin{abstract}
Thermal imaging is an emerging, low-risk modality for early breast cancer decision making without injecting any form of energy into the body. To reduce the burden on radiologists, use of automation is suggested to facilitate better interpretation of thermal images. Hotspots are segmented in the thermograms by k-means clustering algorithm. The features of left and right breasts are more correlated for normal cases and are less correlated for abnormal patients. The investigation results reveal that significant difference in feature values of left and right breast of an abnormal patient is depicted by first order statistical features, namely, kurtosis and variance.
\end{abstract}

\section{Introduction}

Breast Cancer is the leading cause of death among Indian women with rates as high as 25.8 per 100,000 women and mortality 12.7 per 100,000 women. Statistics [4] say that 1 out of 8 Indian women are afflicted by it during their life and it is predicted that 76,000 Indian women may die of the disease every year by 2020 . The most disappointing aspect of breast cancer treatment as a public health concern has been the failure of early screening. It is a societal challenge and delivering an affordable solution to its prevention will benefit society directly. If breast cancer is detected when tumor size is less than $10 \mathrm{~mm}$, the patient has an $85 \%$ chance of cure [4]. Early detection is possible only when the screening test can be performed on the asymptomatic and symptomatic population for routine checkups. Therefore, it is essential to address new research on early detection that is cost effective and has advantages over the gold standard of mammography.

Neo-angiogenesis [26] is the formation of new blood vessels that develop to feed cancerous tumors. This leads to high vascularity which is detectable by thermal imaging. Thermal camera is analogous to a thermometer for breast health assessment that detects functional changes due to the presence of mass. Neoplasms, infections or cancer increases the breast temperature by 1 or $2^{\circ} \mathrm{C}$. Temperature findings are correlated with structural changes. Current outcomes of breast thermography show inconsistent results due to a variety of patterns for different patients [26]. There is insufficient evidence for the use of thermography for detection of breast cancer. The major gaps in knowledge at this time can only be addressed by large-scale, prospective randomized trials. With the incorporation of the latest technology, cheaper yet fast thermal cameras are available that can add curative potential to breast cancer in the next 5 years.

\section{Methods for diagnosis of breast cancer and their limitations}

Over the last few decades, the need for cheap and effective diagnostic techniques to screen and diagnose breast cancer has led to the development of various new technologies. Images are an important documentation in the medical field. Following are the methods used to diagnose abnormalities of the breast:

\subsection{Mammography}

Mammography is an imaging modality that captures 2 projections of each breast - craniocaudal view (CC) and mediolateral oblique view (MLO). It can detect cancer development at the 12th month when the tumor is beyond $1 \mathrm{~cm}$ diameter and X-rays can pass through it, which in many cases have already metastasized. Mammograms can be uncomfortable and traumatic because they often involve high compression of the breast tissue between two plates to get better contrast between non-cancerous and cancerous cells. It shows calcification. The mammogram sensitivity is higher for older women (age 60-69) at $85 \%$ as compared with younger women ( $<50$ years) at $64 \%$. Due to the morphologic similarity, it cannot separate malignant and benign tumors. It also ionizes radiation in the patient and requires high quality, expensive equipment. Pregnant women cannot undergo mammography. False positives are detected in $70 \%$ of the detected areas of concern which triggers emotional stress in patients and results in invasive and avoidable biopsies. Dr. Russell Blaylock, MD, proposes that the likelihood of breast cancer raises $2 \%$ each year a mammogram is performed.The National Cancer Institute $(\mathrm{NCl})$ has stated that for every 15 cases of breast cancer mammography identified, it may actually cause 75 breast cancer diagnosis [4]. Also, due to the 42 pound of pressure, the encapsulation around a tumor can get ruptured and release malignant cells into the bloodstream. 


\subsection{Ultrasound}

Ultrasound or sonography uses sound waves to detect and characterize tumors. Since no radiation is involved, it is a preferred method for screening pregnant women and younger women with dense breasts. It can distinguish between cysts and solid masses. It can neither detect tumors at deeper locations nor identify microcalcifications. The efficiency of ultrasound depends on the expertise of the physician interpreting the image. It is relatively inexpensive and convenient to the patient. It is often used in adjunct with mammography to identify the exact area of suspicion.

\subsection{Breast MRI}

It is a non invasive imaging technique that uses a powerful magnetic field of strength $1.5 \mathrm{~T}$ and provides the highest quality breast images. It can show the smallest of lesions that are not visible in earlier two methods. But, it gives many false positives and is a costly exam. Breast MRI cannot detect micro-calcifications and cannot distinguish between cancerous and noncancerous abnormalities. Pregnant women are not recommended to have breast MRI since a powerful magnet and a contrast agent is used, which produces allergic side effects.

\subsection{Thermography}

Unlike mammography, breast thermography is completely non-invasive, passive, private, contactless and exposes the patient to no radiation hazard. It is painless, because it is simply an image of the patient's breasts. All objects above zero Kelvin emit infrared radiation. According to the Stefan-Boltzmann Law, the radiation emitted by an object is directly proportional to the object's vicinity, emissivity, and the fourth power of its absolute temperature [1]. The measured infrared radiation emitted by one point of the skin can be converted directly into a temperature value that represents this point and then map it to a pixel in a false-color image of the scene. Clinical interpretation of breast thermograms is based on color analysis. Healthy breasts appear purple indicating low heat levels and spots appearing red, orange, or yellow in a thermoscan should be looked at further as these colors may indicate the presence of cancer. Humans are homeotherms, who are capable of maintaining a constant temperature, which is different from that of the surroundings. Warmer areas emit more heat than cooler ones. This military technique was first applied to mammary glands clinically in 1957 to pick changes in blood perfusion [2]. In 1982, Food \& Drugs Administration (FDA) approved thermal imaging to be used in conjunction with mammography for breast cancer detection [5]. Thermography is a functional test and a preventive process, which can be used by women from the age of 23 , unlike mammography which is recommended after the age of 40 . An abnormal thermogram can be a significant biological risk marker in younger women under 40 for existence or continuous development of breast tumor and provides a specific area of breast that needs close examination. It is capable of screening hard to reach areas like upper chest and axilla.

\subsubsection{Breast Thermography : Biological Rationale}

Human body emits part of its own thermal energy in the form of infrared radiation. Skin temperature pattern demonstrates consistent bilateral symmetry; any deviation from the normal is a good evidence of clinical abnormality, indicating metabolic and circulation changes. This is the underlying philosophy of using thermography as a screening tool to diagnose breast cancer. Cancer tissues metabolize faster than other tissues, the heat produced in this process is conveyed to the skin surface, which hints at a possible malignancy or thermally active, fast growing tumor [2]. Also, due to excessive regional vasodilatation caused by nitric oxide (NO) originating from cancerous lesion, there is an increased supply of nutrients and oxygen to the tumour. The tumour location on the breast will have a higher temperature compared to the surrounding normal tissue. Studies from literature show that this temperature difference helps to identify an underlying tumour. The thermal camera gives the temperature distribution over the surface of the skin in the form of an image. Research shows that women with asymmetric thermograms have 10 times higher risk of developing breast cancer than those who had symmetric thermograms [1]. The primary advantage of thermography lies in its efficiency for women with dense breasts and in detecting non palpable breast cancer. It is capable of detecting cancer even before the patient is symptomatic. It is a fast, economic and risk-free process and provides dynamic information of tumor, if screened at regular intervals.

The NoTouch BreastScan (NTBS) machine [22] developed in the USA by UE LifeSciences uses two infrared cameras with a thermal sensitivity of $50 \mathrm{mK}$, each directed to one breast. It uses Artificial Neural Networks to identify the salient features and compare them with known heat patterns typical for tumors. However, at the American Society of Breast Surgeons' annual meeting in Phoenix on May 4, 2012, Dr. C.M. Guilfoyle, a researcher at Bryn Mawr Hospital in Pennsylvania concluded that in a study of about 180 women who had biopsy proven breast cancer, NTBS unit missed about $50 \%$ of cancers!

\section{Literature Review}

\subsection{Processing and Segmentation of Suspicious Region}

Denoising \& Preprocessing: Image quality is a measure of degradation in the image. Noise and artifacts are introduced in the image during compression, image acquisition, storage, etc. Thermal images have a lack of texture and relatively 
low contrast [24]. Variability in acquisition method, in camera handling, introduces variation in quality of images such as varying brightness or orientation in the images. The abnormality detection using asymmetry analysis is complex due to low contrast, low signal to noise ratio and absence of clear edges. Interpreting thermograms requires meticulous training. The fault of high false positive rates of thermogram lies in misinterpretation of the thermogram, rather than the thermogram itself. Bryan F. Jones [3] presented a study about reappraisal of the use of infrared thermography as an indicator of physiological dysfunctions. The results obtained lead to the development in camera technology and in new software development to analyze images statically and dynamically.

Segmentation: The use of abnormal hotspots for feature extraction results in achieving high accuracy in differentiating malignant and benign tumors when compared to whole breast features. Localising and segmenting the tumor spatially in a thermal image is essential for accurate classification of malignancy. Segmentation is a difficult task as it depends on the distance from which the breast thermogram is captured, the image height, the breast size, image background, presence of noise etc. The thermograms are usually acquired in a larger area, which makes the use of segmentation techniques necessary for region of interest (ROI) extraction. Segmentation is also done to separate the right and left breast. Different methods of segmentation such as region-based, threshold-based and edge-based have been exploited in the literature studied. Canny edge detector, Sobel edge detector, Hough transform, C fuzzy techniques are a few to name. Segmentation of thermograms for the identification of breast cancer using projection profile approach is proposed in [19]. In [10], the breast tissue masses were identified into hypoechoic and cystic masses for 60 patients suspected of breast tissue disease. But it was unable to identify iso-echo and calcified mass and had weaknesses in detecting the exact location of the mass. de Oliveira et al. in [9] used automatic segmentation on 180 thermal images; but they used only one side lateral breast images to conduct their work. Thus, their work was studied on a limited basis in clinical trials.

\subsection{Boundary Detection, asymmetry analysis, feature extraction}

Subjectivity is introduced by thermographers who interpret the thermal images. In this regard, automatic feature extraction and $\mathrm{Al}$ algorithms can provide quantifiable indicators to aid in the classification of breasts with and without cancer.

In 2000, Head and Lipari et al. [13] isolated the breasts manually before the analysis. Infrared index was used to quantify six individual asymmetric abnormalities namely, small hot spot, large hot spot, global heat, vascular heat, areolar heat and edge heat by adding individual scores for each abnormality. They compared infrared images of 220 patients who were screened with both first and second generation IR technology. An analysis of their family history, previous hormone therapy and previous biopsy of breast were assessed and results indicated that there was no correlation of these factors with infrared results. Their results pointed out that $35 \%$ of patients had abnormal IR images more than a year before they got diagnosed in mammography, which indicated that IR imaging is a risk marker. Qi, Hairong et al. [6] used Canny edge detector and Hough Transform, to derive the edges and recognize the four feature curves, respectively. Testing images were obtained using the Inframetrics 600M camera provided by Elliott Mastology Centre. Bezier splines were used to view brightness distribution (256 brightness levels). Two curvature curves from two histograms were compared using a threshold based on testing images to decide an indication of asymmetry. The results obtained from 3D histogram of thermal distribution clearly showed the difference between a cancerous image and a non-cancerous image. To attain more accurate classification, supervised pattern classification on a larger database was suggested.

In [8], Kuruganti and Qi measured the asymmetry between left and right breast thermogram by performing feature extraction and using high order statistics like mean, variance, skewness, kurtosis, correlation, entropy, and joint entropy to quantify the distribution of different intensities in each breast. They suggested that the high order statistics (variance, skewness, and kurtosis) were most useful features to detect asymmetry while low-order statistics mean and entropy could not signify any asymmetry. The asymmetry identification was done using two methods: k-means clustering (unsupervised learning) and k-nearest neighborhood (supervised learning) based on feature extraction. They used a small dataset of 6 normal and 18 breast cancer patient thermograms to evaluate the performance of their method. Scales et al. [14] acquired twenty-one 128x128 8bit grayscale thermal images and preprocessed them by first identifying outside boundaries of the breast. Edges were detected by applying the Canny edge operator and were further trimmed. The breast boundary is found by applying the morphological operators to the edge detected image by considering the breast shape of two convex or a smallest ellipse. Edge detection gave false results in the case of the flat lower part of the breast. It was concluded that more robust and intelligent edge detection methods need to be devised to identify regions of interest. The study in [16] involved digital thermography screening of both the breasts of 1008 female patients of age 20-60 years that had not been diagnosed with cancer of breast earlier. Based on the measured temperature gradients $(\Delta \mathrm{T})$ and asymmetry analysis in thermograms, the subjects were classified into one of the three groups, normal $(\Delta \mathrm{T} \leq$ $2.5)$, abnormal $(\Delta T>2.5,<3)$ and potentially having breast cancer $(\Delta T \geq 3)$. It showed sensitivity of $97.6 \%$, specificity of $99.17 \%$, positive predictive value of $83.67 \%$ and negative predictive value of $99.89 \%$. 


\subsection{Using Artificial Neural Networks, Classifiers and Fuzzy Logic}

The extracted features are used as inputs into the classification algorithms to analyze the breast thermal images. Since human eyes cannot perceive the colors in thermograms perfectly, hence to classify suspicious regions in thermograms, into benign and malignant and maximise accuracy, artificial intelligence has been the latest area of interest among many researchers. Determining a suitable combination of features to compose a feature vector is essential for obtaining high precision as too many features pose the problem of overfitting the model. Using only a few features reduces the complexity of models, requires less time and is easier to understand.

Qi and Head [7] proposed an approach that included automatic segmentation (unlike study carried out [13]) and pattern classification of the thermograms to obtain the most relevant features. Abnormalities in the temperature distribution are identified by plotting the thermal histogram of the breast regions (left and right). Unsupervised learning is used and each pixel is relabeled to a certain cluster. Finally, pixel distribution of each cluster for each breast was analyzed, and abnormalities were determined. The selected feature matrix was fed into the classification algorithm [9, 23] to detect TH and classify thermal images into five categories TH1-TH5 namely, TH1- normal uniform non-vascular, TH2vascular ordinary uniform, TH3- vague (questionable), TH4- abnormal, TH5-extremely abnormal, based on the combined vascular and temperature patterns across the two breasts. Francis et al. [15] obtained thermograms of 24 individuals without breast cancer and 12 individuals with breast cancer at steady state and after a cooling period. They extracted 17 different features of the images, consisting of first and second order statistics and texture features. Eventually, the authors reduced the number of features to only four using Principal Component Analysis. They used a Support Vector Machine to classify between breasts with and without tumor. The classifier achieved sensitivities of $83.3 \%$ and $66.7 \%$ post steady state and post cooling thermograms and a specificity of $83.3 \%$ and $75 \%$, respectively.

Gerald Schaefer et al. [11] performed breast cancer analysis using statistical features like moments, histogram features, cross co-occurrence matrix, mutual information and Fourier analysis extracted from the thermograms. The bilateral differences between left and right breast areas are used as a quantifying parameter into a fuzzy rule-based classification system for analysis. A classification accuracy achieved is about $80 \%$, which is limited. Segmentation and classification using K-Means, Fuzzy C-Means (FCM) and Gaussian Mixture Model - Expectations Maximization (GMM-EM) was employed in [21] to segment the IR breast images and their results are compared. The qualitative analysis indicates that the FCM segmentation gives good accuracy and indication of the disease. The results confirmed that thermography is a complementary method for diagnosis. Reference no. [17] studied the problem of subjective interpretation of breast thermograms. It was proposed that the thermograms should be taken within the recommended screening period, classified and analysed in conjunction with an artificial neural network (ANN). Qualitative interpretation of thermal images was carried out using an active contour algorithm. The segmented $256 \times 200$ pixel image was input to the ANN. Then, the thermograms were classified with an accuracy of $59 \%$, sensitivity of $54 \%$, a specificity of $67 \%$, positive predictive value (PPV) of $74 \%$ and negative predictive value (NPV) of $46 \%$. Both benign and malignant processes increase local vascularity and cause variations in temperature, thus limiting the specificity of thermography.

\section{Methods \& Materials}

Interpreting 400,000 color values in thermograms and to diagnose breast abnormality is a huge cognitive overload to the radiologist. Use of automation is suggested in this paper, for better interpretation of thermal images and thus improve the overall accuracy of diagnosis. For the purpose of this study, we used anonymized data of 22 patients provided by Octa Heriena from his past work.

\section{Segmentation \& Asymmetry Analysis}

This stage involves separating the left and right side of the breast by edge detection and setting a threshold using histogram to remove background. K-means clustering is used to find ROI patches i.e high temperature areas. The line profile of an image is a $2 \mathrm{D}$ profile of a line drawn across the image that compares curves of both breasts (mirror site) w.r.t same line to see asymmetries.

II. A. Feature extraction from separated breast images is done using statistical parameters like kurtosis, skewness, mean, standard deviation, variance, entropy, joint entropy, contrast, correlation from GLCM. abnormal.

B. Histogram matching from left and right breasts (gray and RGB histograms) to classify as normal and

Steps for breast segmentation for region of interest (ROI) are as follows :

1. Split image in half into 2 images at centre column

2. Manually crop to discard the irrelevant regions like neck portion, area underneath the breast 

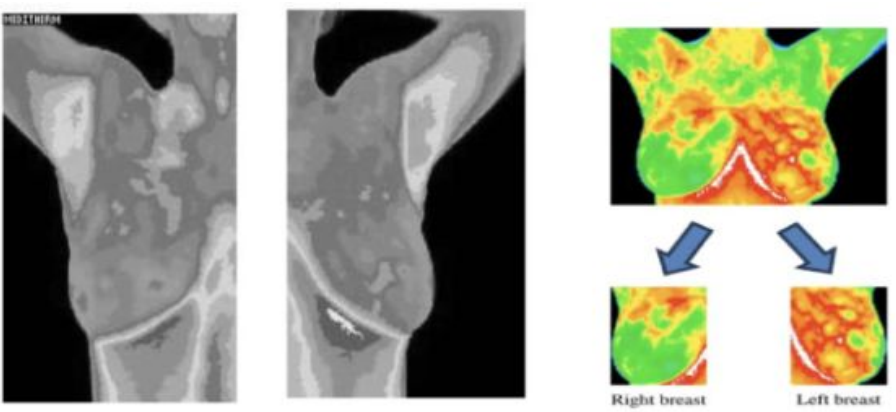

Fig. 1: Segmented right and left breast

5.1 Line Profile of Image : It is a 2D profile of a line drawn across the image that compares curves of both breasts wrt the same line to see asymmetries of mirror sites.
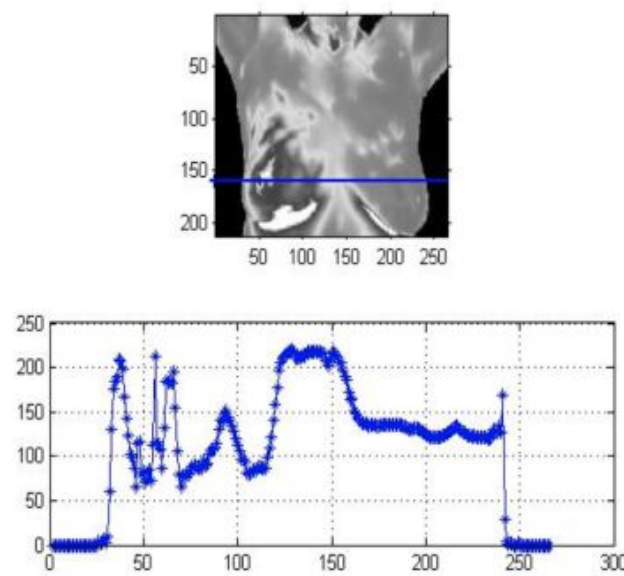
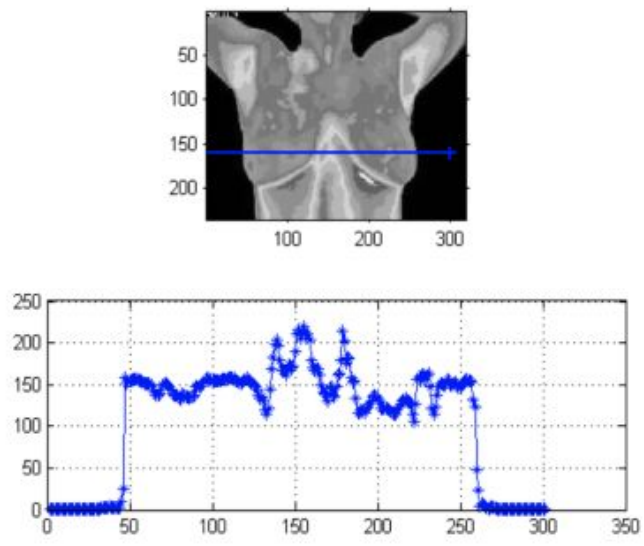

Fig. 2: Line profile of (a) abnormal (asymmetric) and (b) normal patient thermogram (almost symmetric)

The results are treated as a 'Normal' case if the hotspot does not appear in any of the breasts OR it is present in both the breasts symmetrically. If the hotspot is present in areas like armpit, neck and inflammatory folds where the temperature is naturally expected to be more, then also it is considered as 'Normal'. The thermogram is considered as 'Abnormal' if the hotspot appears only in any one of the breasts.

\subsection{Histogram matching}
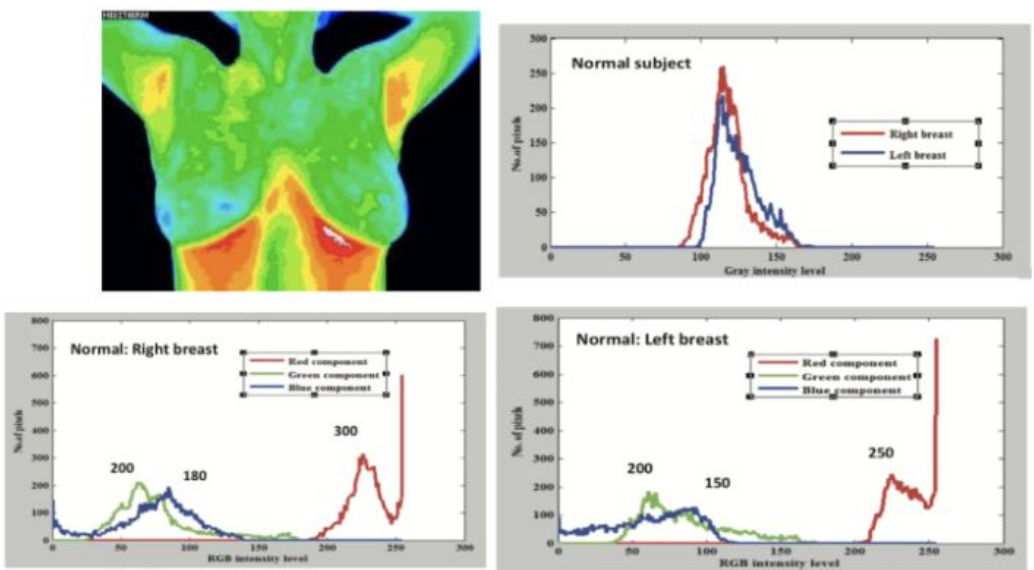

Fig. 3 : (clockwise) normal thermogram, gray histogram, LB and RB of normal patient thermogram 


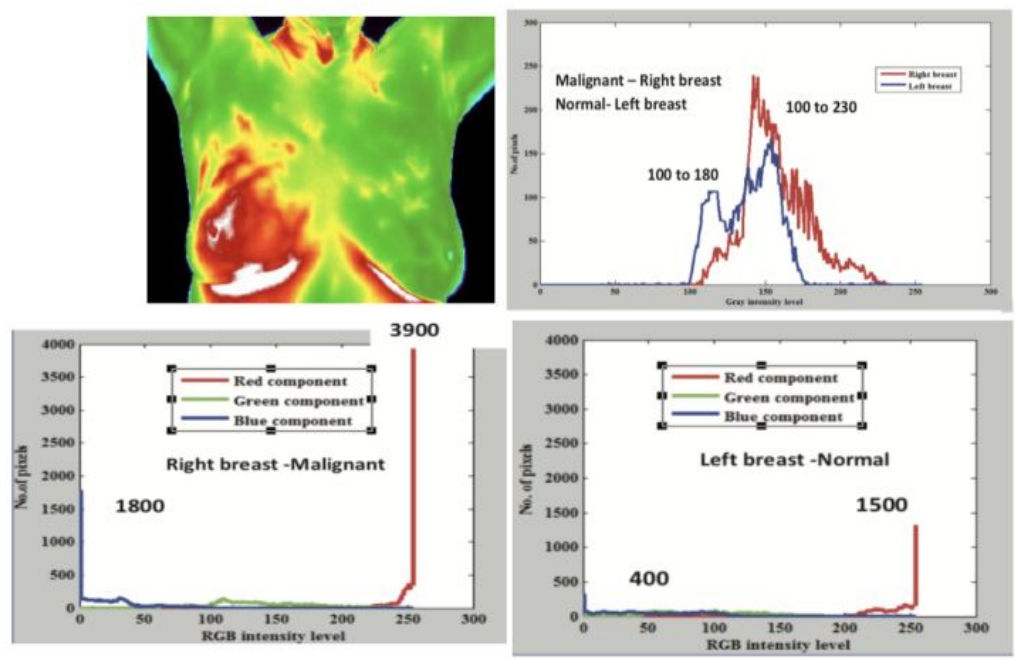

Fig. 4 : (clockwise) abnormal thermogram, gray histogram, LB and RB of abnormal patient thermogram

\subsection{K-Means Clustering for color based segmentation of hotspot}

Clustering technique partitions the pixels of the image into a predefined $\mathrm{k}$ number of disjoint clusters. K-means is an unsupervised classification algorithm. In the K-means algorithm, each cluster is represented by changing cluster centroids, starting from some initial values. K-means clustering determines the distances between the data points and assigns inputs to the nearest cluster. In our case, image pixels are clustered into three clusters: $R, G$ and $B$.

RGB to $L^{*} a^{*} b^{*}$ : The $L^{*} a^{*} b^{*}$ color space enables us to quantify the visual differences between colors. Hence, an RGB image is converted to $L^{*} a^{*} b^{*}$ color space. The $L^{*} a^{*} b^{*}$ space consists of a luminosity layer ' $L^{*}$, chromaticity-layer ' $a *$ indicating where color falls along the red-green axis, and chromaticity-layer ' $b^{* 1}$ indicating where the color falls along the blue-yellow axis. All of the color information is in the ' $a{ }^{* \prime}$ and ' $b{ }^{* \prime}$ layers. To measure the difference between two colors we have used the Euclidean distance metric. Euclidean distance was calculated from the data point to each cluster centroid. Allocate points to the closest cluster. Repeat until clusters no longer change and compute the best clusters.

$$
J=\sum_{j=1}^{k} \sum_{i=1}^{n}\left\|X_{i}^{(j)}-C_{j}\right\|^{2}
$$

This is the objective minimization function, where $\mathrm{k}$ is the number of clusters, $\mathrm{i}$ iterates over all the intensity values, $\mathrm{j}$ iterates over all the centroids (for each cluster) and $\mu \mathrm{i}$ are the centroid intensities.

Cluster pixels in $\mathbf{a}^{*}$ and $\mathbf{b}^{*}$ layers : K-means clustering treats each object as having a location in space. It finds partitions such that objects within each cluster are as close to each other as possible, and as far from objects in other clusters as possible. We specify the number of clusters to be partitioned as 3 . Randomly assign 3 centroids for each cluster based on the average value of R, G and B pixels. For every pixel in the input image, a label is returned corresponding to a cluster, using which three images for each cluster can be displayed for asymmetry analysis.

Segment the hotspots (red pixels) : Cluster 1 contains the red pixels (hotspots) along with slight yellow pixels. To separate red from yellow pixels, we have used the ' $\mathrm{L}^{*}$ layer in the $\mathrm{L}^{*} \mathrm{a}{ }^{*} \mathrm{~b}^{*}$ color space since it contains the brightness values of each color. Extract the brightness values of the pixels in cluster 1 and threshold the yellow pixels to 0 . Thus, we get rid of yellow pixels from the mask and only red hot spots remain. 


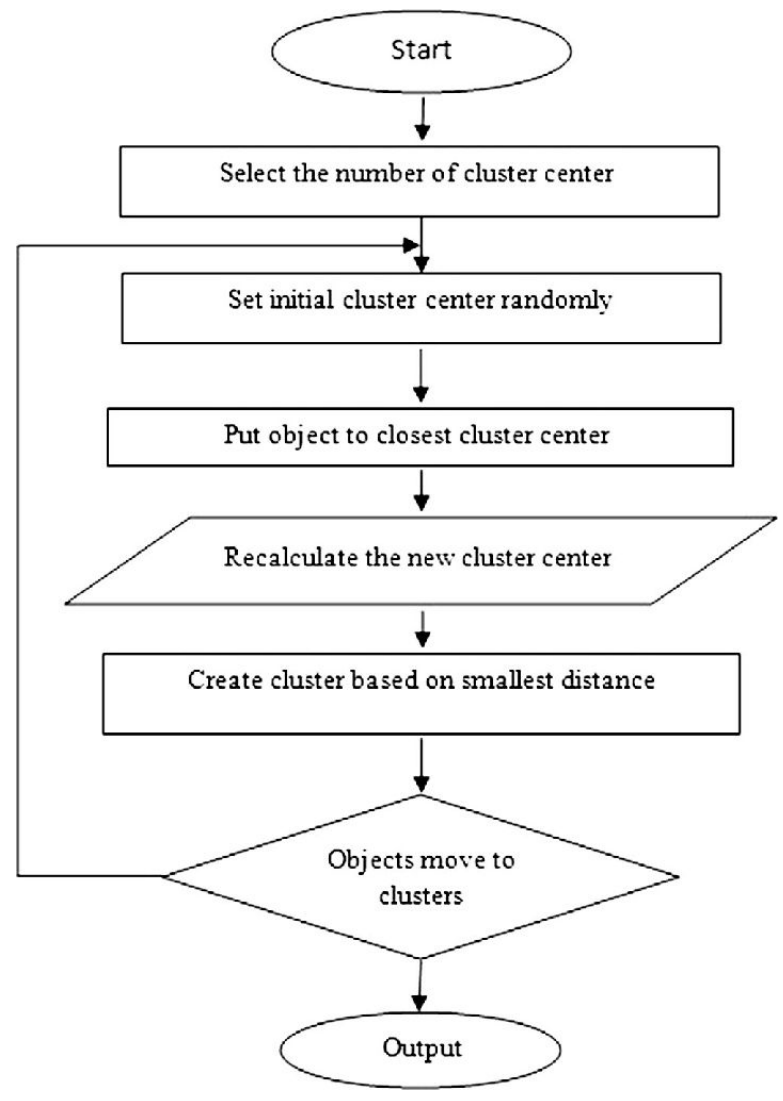

Fig. 5. K Means Algorithm



(a) Normal Thermogram

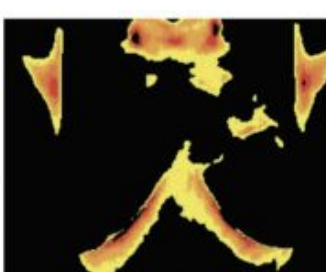

(b) Pixels in cluster 1

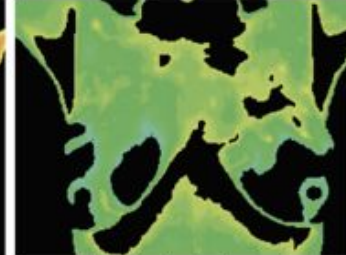

(c) Pixels in cluster 2

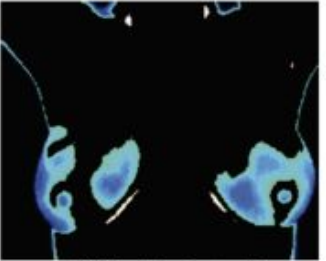

(d) Pixels in cluster 3

Fig. 6. $\mathrm{K}$ means clustering of a normal patient thermogram

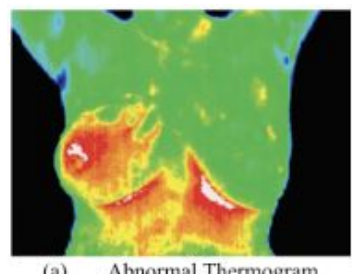

(a) Abnormal Thermogram

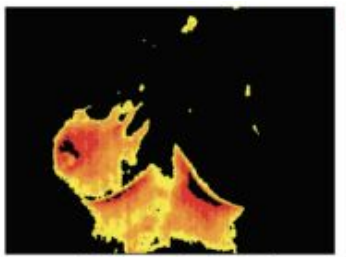

(b) Pixels in cluster 1

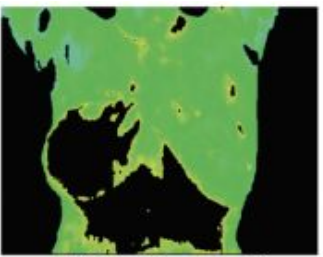

(c) Pixels in cluster 2

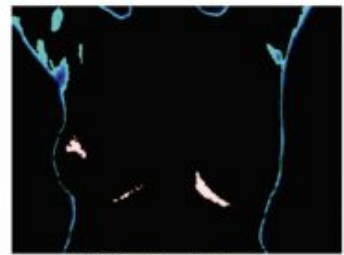

(d) Pixels in cluster 3

Fig. 7. K means clustering of an inflammatory cancer patient thermogram 


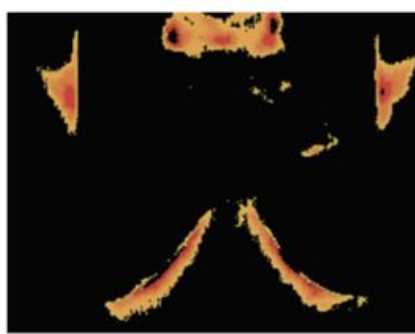

(a) Symmetric hotspots (normal)

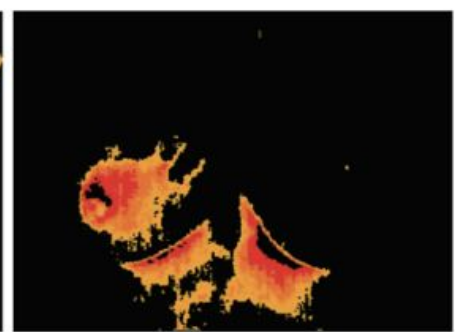

(b) Asymmetric hotspots (cancerous)

Fig. 8. Hot region segmentation of thermograms

\section{Statistical analysis of breast thermograms}

Thermal imaging is a functional imaging system and does not give any anatomical information. Hence, statistical features are used instead of structural features. There are two types of statistical features, namely, first order and second order. First order features do not consider pixel's relationship with neighbourhood pixels. They are computed from intensity histogram. Mean, variance, standard deviation, skewness, kurtosis and entropy are first order statistical features. Second order features deal with the texture of an image and the relative positions of gray levels in an image and consider the relationship between a pixel and its neighbors. They are found from gray-level co-occurrence matrix (GLCM). Energy, entropy, contrast and dissimilarity are second order features. From the obtained results, it can be concluded that first order features are more effective to detect asymmetry than second order features. By analyzing the left and right breasts' intensity distributions of breast thermograms, it is possible to predict the presence of an abnormality in thermograms and categorize them into normal and abnormal.

\section{First Order Statistical Features [25]:}

[a] Mean : It is the first color moment and can be interpreted as the average color in the image.

[b] Standard deviation : It is the second color moment, which is obtained by taking the square root of the variance of the color distribution.

[c] Skewness: Skewness is the third color moment. It measures how asymmetric the color distribution is about the mean, and thus it gives information about the shape of the color distribution.

[d] Kurtosis: Kurtosis is the fourth color moment, and, similarly to skewness. More specifically, kurtosis is a measure of how flat or tall the distribution is in comparison to normal distribution.

Table 1.: List of first order statistical features

\begin{tabular}{|l|l|l|l|}
\hline $\begin{array}{l}\text { Moment } \\
\text { number }\end{array}$ & Name & Measure of & Formula \\
\hline 1 & Mean & Central tendency & $\bar{X}=\frac{\sum_{i=1}^{N} X_{i}}{N}$ \\
\hline 2 & $\begin{array}{l}\text { Variance } \\
\text { (Volatility) }\end{array}$ & Dispersion & $\sigma^{2}=\frac{\sum_{i=1}^{N}\left(X_{i}-\bar{X}\right)^{2}}{N}$ \\
\hline 3 & Skewness & $\begin{array}{l}\text { Symmetry (Positive or } \\
\text { Negative) }\end{array}$ & Skew $=\frac{1}{N} \sum_{i=1}^{N}\left[\frac{\left(X_{i}-\bar{X}\right)}{\sigma}\right]^{3}$ \\
\hline 4 & Kurtosis & Shape (Tall or flat) & Kurt $=\frac{1}{N} \sum_{i=1}^{N}\left[\frac{\left(X_{i}-\bar{X}\right)}{\sigma}\right]^{4}$ \\
\hline
\end{tabular}

\section{Second Order Statistical Features [25]:}

They are computed from the Gray Level Co-occurrence matrix by considering the spatial relationship of pixels. 
[a] Contrast : It is used to measure the local variations present in an image. Images with sharp edges have high contrast.

[b] Energy : It is also known as uniformity of Angular Second Moment (ASM) which is the sum of squared elements from the Grey-level Co-occurrence Matrices (GLCM). It measures the number of repeated pairs. Energy is high if the occurrence of repeated pixel pairs is high.

[c] Homogeneity : It is alternatively called Inverse difference moment, which measures the distribution of elements in the GLCM with respect to the diagonal. Value ranges from 0 to 1 . Value 1 is for the diagonal of GLCM.

[d] Entropy : It measures statistical randomness. It is a useful measure to detect asymmetries. The more equal the temperature distribution, entropy value remains the same. For asymmetric breasts, the difference in entropy is more.

[e] Correlation : It shows the relation between inter-pixels and measures joint probability occurrence of specified pixel pairs.

$$
\begin{gathered}
\text { Energy }=\sum_{m=0}^{G-1} \sum_{n=0}^{G-1} p(m, n)^{2} \\
\text { Entropy }=\sum_{m=0}^{G-1} \sum_{n=0}^{G-1} p(m, n) \log p(m, n) \\
\text { Contrast }=\frac{1}{(G-1)^{2}} \sum_{m=0}^{G-1} \sum_{n=0}^{G-1}(m-n)^{2} p(m, n) \\
\text { Homogeneity }=\sum_{m=0}^{G-1} \sum_{n=0}^{G-1} \frac{p(m, n)}{(1+|m-n|)} \\
\sum_{m=0}^{G-1} \sum_{n=0}^{G-1} m n p(m, n)-\mu_{x} \mu_{y} \\
\sigma_{x} \sigma_{y}
\end{gathered}
$$

where:

$$
\begin{gathered}
\mu_{x}=\sum_{m=0}^{G-1} m \sum_{n=0}^{G-1} p(m, n)^{\prime} \\
\mu_{y}=\sum_{n=0}^{G-1} n \sum_{m=0}^{G-1} p(m, n)^{\prime} \\
\sigma_{x}=\sum_{m=0}^{G-1}\left(m-\mu_{x}\right)^{2} \sum_{n=0}^{G-1} p(m, n)^{\prime} \\
\sigma_{y}=\sum_{n=0}^{G-1}\left(n-\mu_{y}\right)^{2} \sum_{m=0}^{G-1} p(m, n)^{\prime}
\end{gathered}
$$

where, $\mathrm{G}$ is the grey level of the breast image.

$p(m . n)$ is the Grey-level Co-occurrence Matrix (GLCM).

\section{Results \& Discussion}

The results in Table 2. depict that the feature values of left and right breast of a normal breast thermogram are quite similar while, in abnormal breast thermogram (asymmetry) cases, there is a significant difference in the feature values of both breasts. As seen from the plots in Fig. 9, statistical features such as skewness, kurtosis, and variance show a noticeable difference between the left and right breast of an abnormal breast thermogram compared to second order features. 
Table 2.: First and second order statistical features of thermograms (post segmentation)

\begin{tabular}{|c|c|c|c|c|c|}
\hline & & \multicolumn{2}{|c|}{ NORMAL PATIENT } & \multicolumn{2}{|c|}{ ABNORMAL PATIENT } \\
\hline & Features & $\begin{array}{c}\text { Right breast } \\
\text { (frontal view) }\end{array}$ & $\begin{array}{c}\text { Left breast } \\
\text { (frontal view) }\end{array}$ & \begin{tabular}{|c|} 
Right breast \\
(frontal view)
\end{tabular} & $\begin{array}{c}\text { Left breast } \\
\text { (frontal view) }\end{array}$ \\
\hline \multirow{5}{*}{$\begin{array}{c}\text { First order statistical } \\
\text { features }\end{array}$} & Mean & 120.8 & 121.9 & 170.67 & 132.65 \\
\hline & Variance & 161.29 & 108.16 & 413.31 & 108.78 \\
\hline & Standard deviation & 12.7 & 10.4 & 20.33 & 10.43 \\
\hline & Skewness & 0.77 & 0.65 & 0.77 & -0.4 \\
\hline & Kurtosis & 3.76 & 2.98 & 14.23 & 3.39 \\
\hline \multirow{5}{*}{$\begin{array}{c}\text { Second order } \\
\text { statistical features }\end{array}$} & Contrast & 0.2 & 0.23 & 0.34 & 0.23 \\
\hline & Correlation & 0.78 & 0.74 & 0.64 & 0.54 \\
\hline & Energy & 0.32 & 0.35 & 0.26 & 0.19 \\
\hline & Homogeneity & 0.97 & 0.96 & 0.77 & 0.93 \\
\hline & Entropy & 1.34 & 1.27 & 1.84 & 1.69 \\
\hline
\end{tabular}

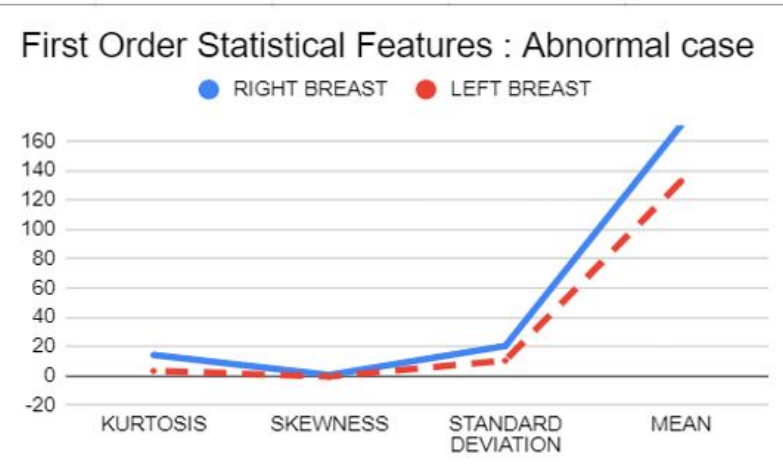

Second Order Statistical Features : Abnormal case

- RIGHT BREAST

LEFT BREAST

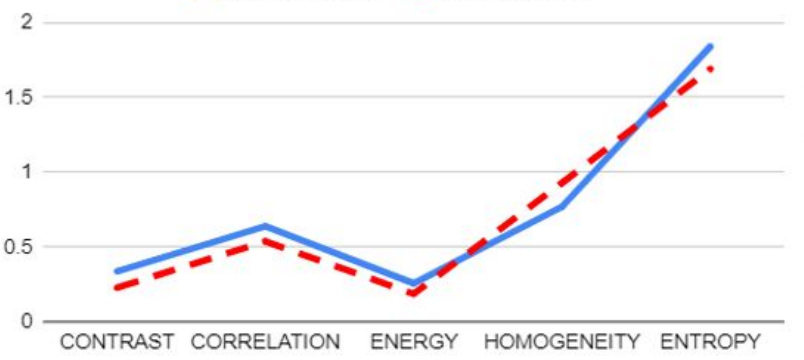

First Order Statistical Features : Normal case

- RIGHT BREAST - LEFT BREAST
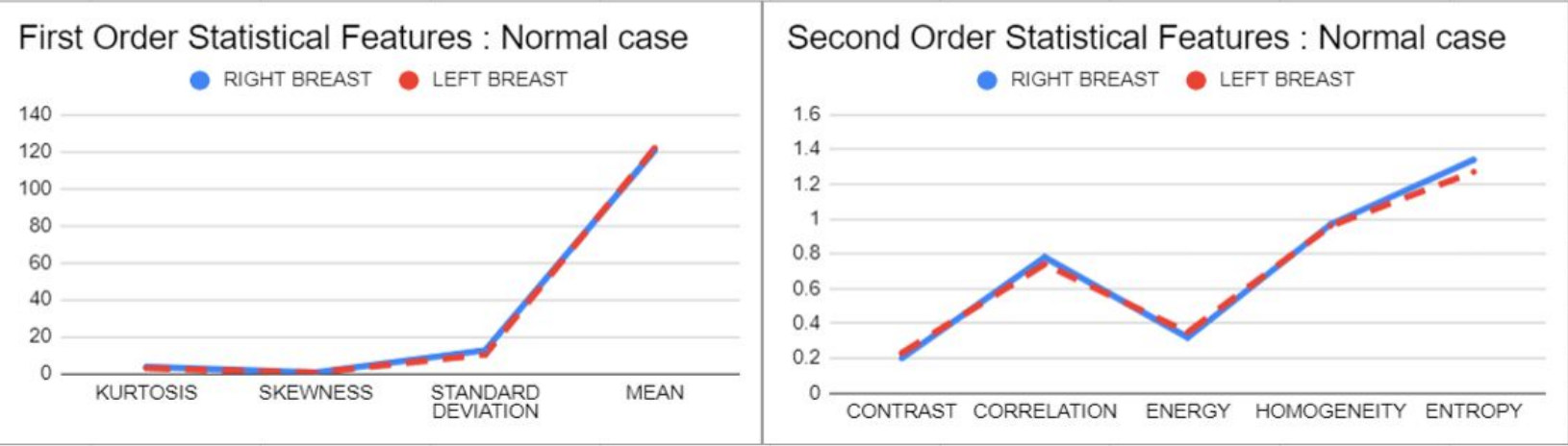

Fig. 9.: First and second order statistical features for a normal and an abnormal case

\section{Conclusion}

Given the rising concern of breast cancer statistics in India, research needs to be done to improve thermal imaging modality for accurate, comfortable and cheaper screening for breast cancer. In this paper, a review of the state-of-the-art methods and techniques used for detecting and classifying the breast cancer using thermography images is discussed. To predict abnormalities, asymmetry analysis is done to understand the patterns. The investigation results reveal that significant difference in feature values of left and right breast of an abnormal patient is depicted by first order statistical features, namely, kurtosis and variance. Thus, this work indicates that thermal imaging is capable of screening breast pathologies by characterization of asymmetry and should be explored further. 


\section{REFERENCES}

[1] R. S. Khandpur, Handbook of Biomedical Instrumentation, Tata McGraw-Hill Education, Second Edition, pp. 670-684, 1994

[2] Joseph D. Bronzino, Medical Devices and Systems, CRC Press, Third Edition, pp. 25-1 - 25-17, 2006.

[3] Jones BF, "A reappraisal of the use of infrared thermal image analysis in medicine". IEEE Transactions on Medical Imaging. vol. 17, no. 6, pp. 1019-27, 1998.

[4] Pink Indian Statistics. Available at: http://www.breastcancerindia.net/statistics/stat_global.html

[5] U.S. Food and Drug Administration. Breast Cancer Screening-Thermography Is Not an Alternative to Mammography: FDA Safety Communication. Available at: https://www.fda.gov/NewsEvents/Newsroom/ PressAnnouncements/ucm257633.htm. Date posted: 6/2/2011. Accessed March 3, 2019.

[6] Qi, Hairong et al. "Detecting breast cancer from infrared images by asymmetry analysis.", Proceedings of the 22nd Annual International Conference of the IEEE, Engineering in Medicine and Biology Society, pp. 1227-1228, 2000.

[7] H. Qi, J.F. Head, "Asymmetry analysis using automatic segmentation and classification for breast cancer detection in thermograms", Proceedings of the 23rd Annual International Conference of the IEEE Engineering in Medicine and Biology Society, vol. 3, pp. 2866-2869, 2001.

[8] P.T. Kuruganti, H. Qi, "Asymmetry analysis in breast cancer detection using thermal infrared images," Engineering in Medicine and Biology, 24th Annual Conference and the Annual Fall Meeting of the Biomedical Engineering Society EMBS/BMES Conference, Proceedings of the Second Joint, vol. 2, pp. 1155-1156, 2002.

[9] de Oliveira, et al. "Segmentation of infrared images: a new technology for early detection of breast diseases," IEEE International Conference on Industrial Technology (ICIT), pp. 1765-1771, 2015.

[10] Iman Zare. et al, "Evaluating the thermal imaging system in detecting certain types of breast tissue masses" Biomedical Research - India, vol. 27, pp. 670-675, 2016.

[11] Schaefer G, Závišek M, Nakashima T, "Thermography based breast cancer analysis using statistical features and fuzzy classification". Pattern Recognition, vol. 42, no.6, pp. 1133-1137, 2009.

[12] Lashkari, AmirEhsan et al. "Full Intelligent Cancer Classification of Thermal Breast Images to Assist the Physician in Clinical Diagnostic Applications" Journal of Medical Signals and Sensors, vol. 6, no. 1, pp. 12-24, 2016.

[13] J. Head, F. Wang, C. Lipari and R. Elliott, "The important role of infrared imaging in breast cancer," IEEE Engineering in Medicine and Biology Magazine, pp. 52-57, 2000.

[14] Scales, Nathan et al. "Automated image segmentation for breast analysis using infrared images." 26th Annual International Conference of the IEEE Engineering in Medicine and Biology Society, vol. 1, pp. 1737-1740, 2004.

[15] Francis SV, et al., "Breast cancer detection in rotational thermography images using texture features," Infrared Physics \& Technology, vol. 67, pp. 490-496, 2014.

[16] Rassiwala, Muffazzal et al. "Evaluation of digital infrared thermal imaging as an adjunctive screening method for breast carcinoma: a pilot study." International Journal of Surgery, vol. 12, pp. 1439-43, 2014.

[17] Ng, E. Y. K. et al. "Statistical analysis of healthy and malignant breast thermography." Journal of Medical Engineering \& Technology, vol. 25, pp 253-263, 2001.

[18] Acct-blog.com. (2019). What is Breast Thermography? - ACCT Blog. [online] Available at: http://acct-blog.com/2009/07/14/what-is-breast-thermography/ [Accessed 16 Apr. 2019].

[19] D. Dayakshini, et al., "Segmentation of Breast Thermogram Images for the Detection of Breast Cancer - A Projection Profile Approach," Journal of Image and Graphics, vol. 3, no. 1, 2015.

[20] Ng, E. Y. K. and N. M. Sudharsan. "Numerical computation as a tool to aid thermographic interpretation." Journal of Medical Engineering \& Technology, vol. 25, no. 2, pp 53-60, 2001.

[21] Prakash, R. Meena et al. "Segmentation of thermal infrared breast images using K-means, FCM and EM algorithms for breast cancer detection." International Conference on Innovations in Information, Embedded and Communication Systems (ICIIECS), pp. 1-4, 2017.

[22] NoTouch BreastScan [Online] Available : http://www.notouchbreastscan.com/index.html

[23] Omranipour, Ramesh et al. "Comparison of the Accuracy of Thermography and Mammography in the Detection of Breast Cancer" Breast Care (Basel, Switzerland) vol. 11, no. 4, pp. 260-264, 2016.

[24] Das, Kakali et al. "Qualitative measures of breast thermograms towards abnormality prediction." 8th International Conference on Computing, Communication and Networking Technologies (ICCCNT), pp. 1-6, 2017.

[25] Computation of Image Central moments: https://itl.nist.gov/div898/handbook/eda/section3/eda35b.htm

[26] Hakim, A., Awale, R.N. Thermal Imaging - An Emerging Modality for Breast Cancer Detection: A Comprehensive Review. J Med Syst 44, 136 (2020). https://doi.org/10.1007/s10916-020-01581-y 